\title{
Efficient Certification and Zero-Knowledge Proofs of Knowledge on Infrastructure Topology Graphs
}

\author{
Thomas Groß \\ School of Computing Science, Newcastle University, UK \\ thomas.gross@newcastle.ac.uk
}

\begin{abstract}
Digital signature schemes are a foundational cryptographic building block in certification and the projection of trust. Based on a signature scheme on committed graphs, we propose a framework of certification and proof methods to sign topology graphs and to prove properties of their certificates in zero-knowledge. This framework allows an issuer, such as an auditing system, to sign the topology representation of an infrastructure. The prover, such as an infrastructure provider, can then convince a verifier of topology properties including connectivity and isolation without disclosing the blueprint of the topology itself. By that, we can certify the structure of critical systems while still maintaining confidentiality. We offer zero-knowledge proofs of knowledge for a general specification language of security goals for virtualized infrastructures such that high-level security goals can be proven over topology certificates. We offer an efficient and practical construction, built upon the Camenisch-Lysyanskaya signature scheme [11], honest-verifier proofs and the strong RSA assumption.
\end{abstract}

\section{Categories and Subject Descriptors}

E.3 [Data]: Data Encryption-Public key cryptosystems

\section{General Terms}

Algorithms, Design, Security

\section{Keywords}

Cloud; topology; digital signatures; graph signature scheme

\section{INTRODUCTION}

Digital signature schemes are foundational cryptographic primitives, in particular to ensure the primary security property of integrity. From their conception [33], digital signature schemes have been employed to sign messages or committed messages [11]. Nowadays, they establish the integrity of systems and their components via certification of software or attestation of software stacks. Their use in attestation of a system, as pursued with Direct Anonymous Attestation (DAA) [8], is particularly relevant when a tenant

Permission to make digital or hard copies of part or all of this work for personal or classroom use is granted without fee provided that copies are not made or distributed for profit or commercial advantage and that copies bear this notice and the full citation on the first page. Copyrights for third-party components of this work must be honored. For all other uses, contact the Owner/Author.

Copyright is held by the owner/author.

CCSW'14, November 7, 2014, Scottsdale, Arizona, USA

ACM 978-1-4503-3239-2/14/11.

http://dx.doi.org/10.1145/2664168.2664175 delegates computation, networking or storage to a provider, such as in outsourcing or cloud computing. In this work, we focus on the question how digital signatures can ensure structural integrity of an infrastructure while maintaining its confidentiality.

The tenants will question the integrity of the systems in which their resources are hosted, in particular, as misconfigurations and insider attackers are considered by ENISA [19] and CSA [17] as high risks exposing the tenants to, e.g., isolation failure. Consequently, cloud security assurance sought to establish structural properties of virtual infrastructures for isolation and deployment patterns [4, 3] as well as for hidden dependency graphs [38, 37]. Our own work with an infrastructure cloud and auditing system provider similarly indicates that structural security properties and a projection of trust from an auditing system to a provider are deemed important.

The systems are typically large topologies with flat hierarchies, by which structural properties, inter-connectivity and isolation are important for the security of the tenants' sub-systems and the system at large. The tenants (and collaborating providers in clouds-ofclouds) will naturally expect the provider to convince them that the system is well-structured and that their own resources are properly isolated from other tenants. However, this fundamental integrity requirement of the tenants is at odds with the confidentiality requirement of the provider. The infrastructure provider naturally requires the blue-print of the infrastructure to be secret. The provider also aims to protect the other tenants from exposure and to ensure that the tenants own confidentiality requirements on their sub-systems are fulfilled. Therefore, we ask: How can a provider convince a verifier that the topology fulfills security properties, such as zone isolation, without disclosing the blueprint of the topology?

We believe that a signature scheme on committed graphs and efficient zero-knowledge proofs of knowledge thereon offers a building block to solve this problem. It provides us with proofs of knowledge that show elaborate statements on topology security properties, while keeping the topology itself confidential. Our work complements existing results in the tenant-verifiable integrity of infrastructures with host-based monitoring [34] and the attestation of physical hosts and virtual machines [8]: Graph signatures offer the confidential attestation of the system structure and enable a provider to convince a verifier that the system is structured securely, while keeping the blueprint of the system secret.

Contributions: 1) We specify the first framework for efficient zero-knowledge proofs of knowledge over signatures on topology graphs, directly applicable to security goals raised by tenants in the domain of virtualized infrastructures expressed in high-level language. 2) The graph signatures and proof systems are a generic solution for arbitrary vertex-/edge-labeled undirected graphs in various problem domains, such as dependency and hierarchy graphs, 
access control policy and provenance graphs or attack trees and structured occurrence nets of incidents. Thus, the scheme has further applications in the cloud, beyond the infrastructure topology itself. 3) We establish general efficient zero-knowledge proofs of knowledge for standard statements over topologies, which allow us to make statements over vertex and edge sets, connectivity and isolation. Combined with known discrete-logarithm based proofs of knowledge [35, 18, 21, 15, 12, 6, 14], we obtain an expressive framework. We believe that the presented topology graph signatures are a suitable building block to close the gap between existing attestation of individual components of the infrastructure and statements on the security of the entire infrastructure. 4) In particular, we propose an efficient general method to prove isolation in linear complexity, while keeping the topology itself confidential. 5) Our system comes with systematic asymptotical and experimental performance evaluation on a prototypical implementation. We are working with an infrastructure cloud and auditing system provider to ensure that the approach is practicable.

The graph signature scheme allows us to bridge the gap between the integrity requirements of the tenant and the confidentiality requirements of the provider in virtualized infrastructures.

\section{SCENARIO AND KEY IDEA}

The key idea of this work is to enable a trusted third-party auditing system to certify a virtualized infrastructure, such that an infrastructure provider can prove to tenants (or other infrastructure providers) that security properties are fulfilled, without disclosing the confidential blueprint of the virtualized infrastructure. Whereas we focus this paper on the tenants, yet see applications for crossprovider proofs as well.

Let us consider the scenario in Figure 1 vis-à-vis the nature of the virtualized infrastructure as a large-scale distributed system, which is dynamically changing through self-provisioning, elastic scalability and provider-issued migrations for optimization of utilization. The sheer scale of an infrastructure will require certification to be partitioned while the dynamics of the system will require that the auditing system is continuously present.

\section{Auditing System.}

We may imagine the auditing system as a flight recorder, which keeps track of the infrastructure state as it observes it. The auditing system analyzes the virtualized infrastructure through the management host interfaces with its own eyes: It employs a topological security analysis tool which comes with its own discovery probes to establish the state of the virtualized infrastructure.

We stress that the audit system can consider arbitrary topology properties such as the dependency graphs investigated by the Structural Reliability Auditor (SRA) [38]. For information flow properties considered here, we find examples of suitable tools in the research domain, e.g., Bleikertz et al. [4, 3], or in industry products, e.g., IBM PowerSC Trusted Surveyor, where both kinds yield a realization model (i.e., a graph representation of the infrastructure's topology). The realization model contains high- and lowlevel components of compute, network and storage resource as well as their connections as described in the management host's configuration. Research in this space had a considerable focus on ensuring that the realization model is a faithful representation of the actual infrastructure. For instance, such a model would contain PortGroups as vertices with associated VLAN identifiers as labels. The auditing system annotates the graph further, for instance with its own geolocation.

From this graph representation of the infrastructure, the auditor issues a graph signature $\sigma$ to the provider: The auditing system certifies the topology and configuration as seen by the discovery probes of the security tool. Given the dynamically changing nature of the virtualized infrastructure, the auditing system will sign a continuous sequence of graphs bound to a time index in short succession. Therefore, we will have a requirement that the issuing can be done rapidly.

\section{Infrastructure Provider.}

Given such a graph signature, the provider can prove to tenants in zero-knowledge that security properties specified in a high-level language, such as VALID [2] or a graph rewriting language, such as GROOVE, are fulfilled. Observe that the graph signatures are generic and independent from tenants and the security properties they ask for. In practice, this is driven by tenant requests, by which the infrastructure provider stores a batch of signatures for a retention period.

\section{Tenant.}

A tenant auditing his own virtualized infrastructure fixes a time index $t$ and asks the provider questions about the topology of the infrastructure at that time, after the fact. Examples include the following questions:

- "Are my resources isolated from any competitor?" (Zone isolation)

- “Are all my resources geolocated in Europe?” (Deployment correctness)

- "Are all my resources reachable by two independent paths?" (Availability)

Upon receiving such a question, the provider can then look up the graph signature for time index $t$, and compute a zero-knowledge proof of knowledge that the required property is fulfilled on the signature without disclosing further information about the infrastructure: "Yes, I hold a graph signature for time index $t$ issued by a trusted auditing system, for which the property holds that your resources are isolated from any competitor." The tenant verifies the zero-knowledge proof with respect to the public key of the auditing system and can, thereby, be convinced that the property was fulfilled in the state the auditing system has observed.

\section{Building Blocks and Outline.}

To establish the topology certification, we need multiple building blocks spanning a range of conceptual levels. First, we need a graph signature scheme that can be employed for a certification by an auditing system. The key idea here is to establish an anonymous credential system that operates on graphs. It must be capable of binding different elements of graphs together (e.g. vertices and their labels) and of making them accessible to zero-knowledge proofs. The preliminaries in $\S 4$ are setting the stage: CamenischLysyanskaya signatures and Camenisch-Groß encoding. Having established the preliminaries, $\$ 5$ introduces the cryptographic interfaces for the graph signature scheme algorithms and the library of proof predicates we construct subsequently.

Second, we need to establish how the signature scheme can be implemented, in particular, how the foundational primitive of a proof of representation works. Thus, the next sections focus on the implementation of the graph signature interfaces, where $\$ 5.2$ establishes the underlying encoding for undirected. \$5.3.1 offers the core building blocks of proofs of representations as well as key generation.

Third, we will investigate how to compile zero-knowledge proofs for specific statements, by which $\S 6$ offers constructions for a li- 


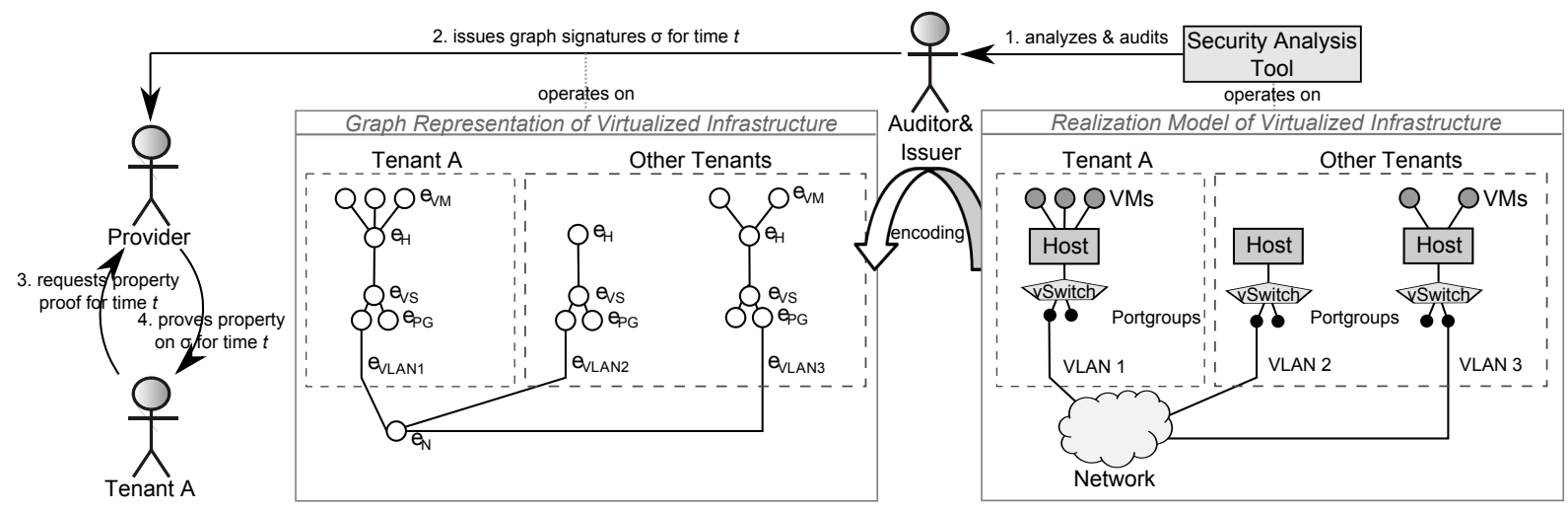

Figure 1: System Model of an auditing system operating on a realization model of a virtualized infrastructure. The auditing system continuously inspects the infrastructure with a security analysis tool at its disposal and certifies graph signatures to the infrastructure provider. The provider, in turn, proves properties of the infrastructure to tenants upon request.

brary of graph proofs, including statements over vertex sets, connectivity and isolation.

Fourth, we need to convince ourselves that a graph signature and proof system can be implemented sufficiently efficiently and scalably, which we investigate in $\$ 7$ asymptotically as well as experimentally. $\$ 8$ compares this work with earlier proposals for transitive and homomorphic graph signatures and zero-knowledge proofs on graphs, while $\$ 9$ discusses future work.

\section{SYSTEM MODEL}

As system model (Figure 1) we consider a virtualized infrastructure, with compute, network and storage resources, operated via management hosts. A typical example of such an infrastructure is VMware administered with vCenter. We consider three parties: an infrastructure provider operating the virtualized infrastructure (acting as recipient of graph signatures and as prover towards tenants), a trusted auditing system with read-only access to the management hosts (acting as issuer of graph signatures) and multiple tenants (acting as verifiers of zero-knowledge proofs of knowledge on graph signatures).

Trust is asymmetric: The provider trusts the auditor to keep the infrastructure configuration confidential; the tenants trust the auditor to sign only well-formed graphs and only as faithfully obtained from the realization model of the security tool. The faithful representation of virtualized infrastructure configurations (and their changes) in graph models has been studied in the recent years starting from Bleikertz et al. [4] and is assumed as given in this work.

Fault Model: We assume hypervisors and management hosts to be correct: Software failures of hypervisors are out of scope; security failures, in particular, both spawning from misconfigurations (non-malicious human faults) and insider attackers (malicious human faults) are in the scope of this work. We stress that even if the virtualized infrastructure uses secure hypervisors and is protected by TPMs, malicious or non-malicious misconfigurations are a major problem $[19,17]$ and have been observed in our own work with industrial partners.

\section{PRELIMINARIES}

\subsection{Assumptions}

Special RSA Modulus. A special RSA modulus has the form $N=p q$, where $p=2 p^{\prime}+1$ and $q=2 q^{\prime}+1$ are safe primes, the corresponding group is called special RSA group. Strong RSA
Assumption [33, 21]. Given an RSA modulus $N$ and a random element $g \in \mathbb{Z}_{N}^{*}$, it is hard to compute $h \in \mathbb{Z}_{N}^{*}$ and integer $e>1$ such that $h^{e} \equiv g \bmod N$. The modulus $N$ is of a special form $p q$, where $p=2 p^{\prime}+1$ and $q=2 q^{\prime}+1$ are safe primes. Quadratic Residues. The set $\mathrm{QR}_{N}$ is the set of Quadratic Residues of a special RSA group with modulus $N$ (cf. [36]).

\subsection{Integer Commitments}

Damgård and Fujisaki [18] showed for the Pedersen commitment scheme [31] that if it operates in a special RSA group and the committer is not privy to the factorization of the modulus, then the commitment scheme can be used to commit to integers of arbitrary size. The commitment scheme is information-theoretically hiding and computationally binding. The security parameter is $\ell$. The public parameters are a group $G$ with special RSA modulus $N$, and generators $\left(g_{0}, \ldots, g_{m}\right)$. In order to commit to the values $\left(V_{1}, \ldots, V_{l}\right) \in\left(\mathbb{Z}_{n}^{*}\right)^{l}$, pick a random $R \in\{0,1\}^{\ell}$ and set

$$
C=\operatorname{Commit}\left(R, V_{1}, \ldots, V_{l}\right)=g_{0}^{R} \prod_{i=1}^{l} g_{i}^{V_{i}} .
$$

\subsection{Known Discrete-Logarithm-Based Proofs}

In the common parameters model, we use several previously known results for proving statements about discrete logarithms, such as (1) proof of knowledge of a discrete logarithm modulo a prime [35] or a composite [18, 21], (2) proof of knowledge of equality of representation modulo two (possibly different) prime [15] or composite [12] moduli, (3) proof that a commitment opens to the product of two other committed values [7, 12], (4) proof that a committed value lies in a given integer interval $[6,12,14]$, and also (5) proof of the disjunction or conjunction of any two of the previous ones [16]. These protocols modulo a composite are secure under the strong RSA assumption and modulo a prime under the discrete logarithm assumption.

Proofs as described above can be expressed in the notation introduced by Camenisch and Stadler [13]. For instance,

$$
P K\left\{(\alpha, \beta, \delta): y=g^{\alpha} h^{\beta} \wedge \tilde{y}=\tilde{g}^{\alpha} \tilde{h}^{\delta} \wedge(u \leq \alpha \leq v)\right\}
$$

denotes a "zero-knowledge Proof of Knowledge of integers $\alpha, \beta$, and $\delta$ such that $y=g^{\alpha} h^{\beta}$ and $\tilde{y}=\tilde{g}^{\alpha} \tilde{h}^{\delta}$ holds, where $u \leq$ $\alpha \leq v$," where $y, g, h, \tilde{y}, \tilde{g}$, and $\tilde{h}$ are elements of some groups $G=\langle g\rangle=\langle h\rangle$ and $\tilde{G}=\langle\tilde{g}\rangle=\langle\tilde{h}\rangle$. The convention is that Greek letters denote quantities of which knowledge is being proven, while 
all other values are known to the verifier. We apply the Fiat-Shamir heuristic [20] to turn such proofs of knowledge into signatures on some message $m$; denoted as, e.g., $\operatorname{SPK}\left\{(\alpha): y=g^{\alpha}\right\}(m)$. Given a protocol in this notation, it is straightforward to derive an actual protocol implementing the proof.

We introduce the following short-hands: i. A modulus statement $(\bmod N)$ in the $P K$-header denotes the default modulus for subsequent non-range proof congruences. ii. An all quantifier $\forall i$ denotes that the secrets/terms in its scope are iterated over $i$. iii. We decompose proofs of knowledge statements in multiple steps and require referential integrity between the secrets of the steps.

\subsection{Camenisch-Lysyanskaya Signatures}

Let us introduce Camenisch-Lysyanskaya (CL) signatures in a Strong RSA setting [11].

Let $\ell_{\mathcal{M}}, \ell_{e}, \ell_{N}, \ell_{r}$ and $L$ be system parameters; $\ell_{r}$ is a security parameter, $\ell_{\mathcal{M}}$ the message length, $\ell_{e}$ the length of the Strong RSA problem instance prime exponent, $\ell_{N}$ the size of the special RSA modulus, $L$ the number of message bases. The scheme operates with a $\ell_{N}$-bit special RSA modulus. Choose, uniformly at random, $R_{0}, \ldots, R_{L-1}, S, Z \in \mathrm{QR}_{N}$. The public key $p k_{1}$ is $\left(N, R_{0}, \ldots, R_{L-1}, S, Z\right)$, the private key $s k_{1}$, the factorization of the special RSA modulus.

The message space is $\left\{\left(m_{0}, \ldots, m_{L-1}\right): m_{i} \in \pm\{0,1\}^{\ell_{\mathcal{M}}}\right\}$. Signing algorithm. On input $m_{0}, \ldots, m_{L-1}$, choose a random prime number $e$ of length $\ell_{e}>\ell_{\mathcal{M}}+2$, and a random number $v$ of length $\ell_{v}=\ell_{N}+\ell_{\mathcal{M}}+\ell_{r}$. Compute

$$
A=\left(\frac{Z}{R_{0}^{m_{0}} \cdots R_{L-1}^{m_{L-1}} S^{v}}\right)^{1 / e} \bmod N
$$

The signature consists of $(e, A, v)$.

Verification algorithm. To verify that the tuple $(e, A, v)$ is a signature on message $\left(m_{0}, \ldots, m_{L-1}\right)$, check that the following statements hold:

$$
Z \equiv A^{e} R_{0}^{m_{0}} \cdots R_{L-1}^{m_{L-1}} S^{v} \quad(\bmod N)
$$

with $m_{i} \in \pm\{0,1\}^{\ell_{\mathcal{M}}}$, and $2^{\ell_{e}}>e>2^{\ell_{e}-1}$.

THEOREM 4.1. [11]

The signature scheme is secure against adaptive chosen message attacks [23] under the strong RSA assumption.

Proving Knowledge of a Signature. A prover can prove that she possesses a CL-signature without revealing any other information about the signature (as well as use the primitives in $\S 4.3$ ). The prover randomizes $A$ : Given a signature $(A, e, v)$, the tuple $\left(A^{\prime}:=\right.$ $\left.A S^{-r} \bmod N, e, v^{\prime}:=v+e r\right)$ is a valid signature as well. Now, provided that $A \in\langle S\rangle$ and that $r$ is chosen uniformly at random from $\{0,1\}^{\ell_{N}+\ell_{\varnothing}}$, the value $A^{\prime}$ is distributed statistically close to uniform over $\mathrm{QR}_{N}$. Thus, the user could compute a fresh $A^{\prime}$ each time, reveal it, and then run the protocol

$$
\begin{aligned}
P K\{ & \left(\varepsilon, \nu^{\prime}, \mu_{0}, \ldots, \mu_{L-1}\right): \\
Z & \equiv \pm R_{0}^{\mu_{0}} \cdots R_{L-1}^{\mu_{L-1}} A^{\prime \varepsilon} S^{\nu^{\prime}}(\bmod N) \wedge \\
\mu_{i} & \left.\in \pm\{0,1\}^{\ell_{\mathcal{M}}} \wedge \varepsilon \in\left[2^{\ell_{e}-1}+1,2^{\ell_{e}}-1\right]\right\}
\end{aligned}
$$

\subsection{Set Membership from CL-Signatures}

Set membership proofs can be constructed from CL-Signatures following a method proposed by Camenisch et al. [9]. For a set $\mathcal{S}=\left\{m_{0}, \ldots, m_{i}, \ldots, m_{l}\right\}$, the issuer signs all set members $m_{i}$ in CL-Signatures $\sigma_{i}=(A, e, v)$ and publishes the set of messagesignature pairs $\left\{\left(m_{i}, \sigma_{i}\right)\right\}$ with integrity. To prove set membership of a value committed in $C$, the prover shows knowledge of the blinded signature $\sigma_{i}^{\prime}$ corresponding to the message $m_{i}$ and equality of exponents with $C$. We describe the implementation in the extended version [26] and denote a set membership proof $\mu[C] \in \mathcal{S}$, which reads $\mu$ encoded in commitment $C$ is member of set $\mathcal{S}$.

\subsection{Camenisch-Groß Encoding}

The Camenisch-Groß (CG) Encoding [10] gives the CL message space structure by encoding multiple binary and finite-set values into a single message, and we will use a similar paradigm to encode graphs efficiently.

The core principle of the CG-Encoding is to represent binary and finite-set attribute values as prime numbers. It uses divisibility and coprimality to show whether an attribute value is present in or absent from a credential. The attribute values certified in a credential, say $e_{i}, e_{j}$, and $e_{l}$, are represented in a single message of the CL-Signature, by signing the product of their prime representative $E=e_{i} \cdot e_{j} \cdot e_{l}$ in an Integer attribute. The association between the value and the prime number of the encoding is certified by the credential issuer.

Divisibility/AND-Proof. To prove that a disclosed prime representative $e_{i}$ is present in $E$, we prove that $e_{i}$ divides the committed product $E$, we show that we know a secret $\mu^{\prime}$ that completes the product:

$$
P K\left\{\left(\mu^{\prime}, \rho\right): \quad D \equiv \pm\left(g^{e_{i}}\right)^{\mu^{\prime}} h^{\rho}(\bmod N)\right\} .
$$

Coprimality/NOT-Proof. We show that one or multiple prime representatives are not present in a credential, we show coprimality. To prove that two values $E$ and $F$ are coprime, i.e., $\operatorname{gcd}(E, F)=$ 1 , we prove that there exist integers $a$ and $b$ such that Bézout's Identity equals 1 , where $a$ and $b$ for this equation do not exist, if $\operatorname{gcd}(E, F)>1$.

$$
\begin{gathered}
P K\left\{\left(\mu, \rho, \alpha, \beta, \rho^{\prime}\right): \quad D \equiv \pm g^{\mu} h^{\rho}(\bmod N) \wedge\right. \\
\left.g \equiv \pm D^{\alpha}\left(g^{F}\right)^{\beta} h^{\rho^{\prime}}(\bmod N)\right\} .
\end{gathered}
$$

OR-Proof To show that a credential contains an attribute $e$ that is contained in an OR-list, we show there exists an integer $a$ such that $a e=\prod_{i}^{\ell} e_{i}$; if $e$ is not in the list, then no such integer $a$ as $e$ does not divide the product. We use the notation $\alpha \subseteq \Xi$ for an OR-proof that $\alpha$ contains one or more values of $\Xi$.

\section{GRAPH SIGNATURE SCHEME}

This section establishes the core graph signature scheme: The key point here is that we offer a graph encoding that lifts the anonymous credential scheme given by the Camenisch-Lysyanskaya signatures $(\S 4)$ to entire graphs as messages. The encoding is special in that it keeps all elements of the graph (vertices, edges, labels) accessible to efficient zero-knowledge proofs.

\subsection{Cryptographic System Interface}

Let us first specify the abstract interface of a graph signature scheme and associated proofs over graph properties. The core signature scheme consists of five algorithms:

Commit(), Keygen(), Sign(), HiddenSign(), and Verify(),

where we consider HiddenSign () and Verify () in the extended version [26], that is, the joint signing of hidden committed graphs.

Commit $(\mathcal{G} ; R)$ is a probabilistic polynomial-time algorithm, providing an Integer commitment lifted to graphs. It takes as input a graph $\mathcal{G}$ encoded with the encoding encode $(\mathcal{G})$ specified in $\S 5.2$ and randomness $R$.

Keygen $\left(1^{\ell}\right.$, params $)$ establishes the key setup for the graph signature scheme in a probabilistic polynomial-time algorithm. It 
takes as input the security parameter $\ell$ and the public parameters of the commitment scheme Commit. It outputs a key pair $(p k, s k)$, where $p k$ is the public key of the issuer and $s k$ is its secret key.

$\operatorname{Sign}\left(p k_{1}\right)$ is a probabilistic polynomial-time algorithm run by issuer I. It signs a graph $\mathcal{G}_{1}$. The public input is a commitment on the user sub-graph issuer's public key $p k_{1}$. The issuer's private input is the graph $\mathcal{G}_{1}$ and his secret key $s k_{1}$. The output is a signature on the graph $\sigma=\sigma\left(\mathcal{G}_{1}\right)$.

In addition, we provide proof predicates for graph signatures (cf. with Table 1): First, we provide predicates for the graph proof of representation possession as well as decompositions to vertex and edge level, both implemented in \$5.3.1. Second, we consider sets of graph elements with set coverage cover, pair-wise disjointness disjoint and partition partition. Third, we have predicates on connectivity edge and connected and its complement isolation isolated $=\neg$ connected. We establish an implementation for zeroknowledge proofs of knowledge thereof in $\$ 6$. These proofs can be combined with known proofs of knowledge based on discretelogarithms and composed to Boolean formulas with logical connectives $\wedge$ and $\vee$.

\section{REMARK 1 (Cloud SECurity ASSurance).}

The predicates introduced in Table 1 correspond to the major predicates of VALID [2], a formal specification language of cloud security goals suitable for automated model checking. VALID expresses goal states as a set (conjunction) of positive and negative facts constrained by a Boolean condition list. It uses terms, such as edge $(\cdot, \cdot)$ or connected $(\cdot, \cdot)$ to express alarm states on topology graphs.

Subsequently, we will introduce the implementations for proofs of knowledge for these different functions successively. \$5.2 introduces the encoding of undirected graphs itself. $\$ 5.3$ introduces the key generation Keygen, the proof of representation graph and the issuing and verification algorithms HiddenSign and Verify. Subsequently, $\$ 6$ contains the constructions for the set and connectivity predicates.

\subsection{Graph Encoding}

The key idea of the graph encoding is this: we represent vertex identifiers and labels as prime numbers. This allows us to bind different elements of the graph together by signing the product of their representations. In addition, we can have efficient proofs of properties such as connectedness and isolation, by arguing over divisibility and coprimality of the prime products. The proofs can be implemented efficiently with discrete-logarithm zero-knowledge proofs.

We consider strict undirected graphs over finite vertex sets and finite sets of vertex and edge labels, where vertices and edges can have multiple labels. We describe the encoding and proofs for directed graphs in the extended version of this paper [26].

$\begin{array}{ll}\mathcal{V} & \text { Finite set of vertices } \\ \mathcal{E} \subseteq(\mathcal{V} \times \mathcal{V}) & \text { Finite set of edges } \\ \mathcal{G}=\left(\mathcal{V}, \mathcal{E}, t_{\mathcal{V}}, t_{\mathcal{E}}\right) & \text { Graph } \\ \mathcal{L}_{\mathcal{V}}, \mathcal{L}_{\mathcal{E}} & \text { Finite sets labels } \\ f_{\mathcal{V}}: \mathcal{V} \rightarrow \mathcal{P}\left(\mathcal{L}_{\mathcal{V}}\right) & \text { labels of a given vertex } \\ f_{\mathcal{E}}: \mathcal{E} \rightarrow \mathcal{P}\left(\mathcal{L}_{\mathcal{E}}\right) & \text { labels of a given edge } \\ n=|\mathcal{V}|, m=|\mathcal{E}| & \text { number of vertices and edges }\end{array}$

We call a prime representative, a prime number which denotes an element of a graph. For each vertex $i$ in $\mathcal{V}$, we introduce a vertex identifier, a prime $e_{i}$, which represents this vertex in credential and proofs. The symbol $\perp$, associated with identifier $e_{\perp}$ represents that a vertex is not present. All vertex identifiers are pair-wise different. We call the set of all vertex identifiers $\Xi_{\mathcal{V}}$, their product
$\chi_{\mathcal{V}}=\Pi \Xi_{\mathcal{V}}$. For each label $k$ in the label sets $\mathcal{L}_{\mathcal{V}}$ and in $\mathcal{L}_{\mathcal{E}}$, we introduce a prime representative $e_{k}$. All label representatives are pair-wise different. We call the set of all label representatives $\Xi_{\mathcal{L}}$, their product $\chi_{\mathcal{L}}=\Pi \Xi_{\mathcal{L}}$. Vertex identifiers and label representatives are disjoint:

$$
\Xi_{\mathcal{V}} \cap \Xi_{\mathcal{L}}=\emptyset \quad \Leftrightarrow \quad \operatorname{gcd}\left(\chi_{\mathcal{V}}, \chi_{\mathcal{L}}\right)=1
$$

\subsubsection{Encoding Vertices and Edges}

To encode a vertex and its associated labels into a graph commitment or CL-Signature, we encode the product of the vertex identifier $e_{i} \in \Xi_{\mathcal{V}}$ and the prime representatives $e_{k} \in \Xi_{\mathcal{L}}$ for $k \in f_{\mathcal{V}}(i)$ of the labels into a single message of the signature. The product of prime representatives is encoded as exponent of dedicated vertex bases $R \in G$ V.

To get a compact encoding and efficient proofs thereon, the encoding needs to maintain the graph structure and to allow us to access it to prove higher-level properties, such as connectivity and isolation. The proposal we make in this paper after evaluating multiple approaches is to use divisibility and coprimality similar to the CG-Encoding to afford us these efficient operations over the graph structure, while offering a compact encoding of edges.

Recall that each vertex is certified with a vertex identifier from $\Xi \mathcal{V}$, e.g., $e_{i}$ or $e_{j}$. For each edge $(i, j) \in \mathcal{E}$, we include an edge attribute as exponent of a random edge base $R_{\pi(i, j)} \in G_{\mathcal{E}}$, containing the product of the vertex identifiers and the associated label representatives $e_{k} \in \Xi_{\mathcal{L}}$ for $k \in f_{\mathcal{E}}(i, j)$ of the edge:

$$
E_{(i, j)}:=e_{i} \cdot e_{j} \cdot \Pi_{k \in f_{\mathcal{E}}(i, j)} e_{k}
$$

DEFINITION 1 (WELL-FORMED GRAPH).

We call a graph encoding well-formed iff 1. the encoding only contains prime representatives $e \in \Xi_{\mathcal{V}} \cup \Xi_{\mathcal{L}}$ in the exponents of designated vertex and edge bases $R \in G_{\mathcal{V}} \cup G_{\mathcal{E}}$, 2. each vertex base $R \in G \mathcal{V}$ contains exactly one vertex identifier $e_{i} \in \Xi_{\mathcal{V}}$, pairwise different from other vertex identifiers and zero or more label representatives $e_{k} \in \Xi_{\mathcal{L}}$, and 3. each edge base $R \in G_{\mathcal{E}}$ contains exactly two vertex identifiers $e_{i}, e_{i} \in \Xi_{\mathcal{V}}$ and zero or more label representatives $e_{k} \in \Xi_{\mathcal{L}}$.

\subsection{Signing Committed Graphs}

Once we have the encoding, the next question is how graphs can be signed. Luckily, the encoding embeds the graph directly into the Camenisch-Lysyanskaya signature scheme, we can employ the signing method given in the preliminaries $(\$ 4)$ directly for the simple case that an audit system inspects a topology and signs the graph it observes.

The full graph signature scheme supports a joint issuing of graph signatures with a hidden committed subgraph contributed by a user and another known subgraph contributed by the issuer. We define the issuing process, including the considerations for hidden graphs being merged in the extended version.

As a point of reference, we give the structure of the graph signatures, as it guides how we prove representation of such signatures. We have seen in the encoding (\$5.2) that it operates over bases $R_{\pi(i)} \in G_{\mathcal{V}}$, which store attributes encoding vertices in the exponent, and bases $R_{\pi(i, j)} \in G_{\mathcal{E}}$, which store attributes encoding edges in the exponent. The base association is randomized by 
Table 1: Proof of knowledge predicates for graph signatures (cf. VALID [2]).

\begin{tabular}{llc}
\hline Predicate & Description & \\
\hline possession $\left(\mathcal{G}, \sigma, \mu_{i}\right)$ & Proof of possession of a graph signature $\sigma$ & $\S 5.3 .1$ \\
vertices $\left(\mathcal{G}, \varepsilon_{i}, \gamma_{i}\right)$ & Proof of composition of graph vertices of a proof of possession & $\S 5.3 .1$ \\
edges $\left(\mathcal{G}, \varepsilon_{i}, \varepsilon_{i}, \gamma_{(i, j)}\right)$ & Proof of composition of graph edges of a proof of possession & $\S 5.3 .1$ \\
$\operatorname{graph}\left(\mathcal{G}, \mu_{i}\right)$ & Proof of representation and well-formedness (extended version [26]) & $\S 5.3 .1$ \\
$\operatorname{set}(\mathcal{V}, V)$ & Representation of a set $V \subseteq \mathcal{V}$ & $\S 6.1$ \\
$\operatorname{cover}\left(\mathcal{V}, V_{1}, \ldots, V_{k}\right)$ & Vertex set coverage $\bigcup\left(V_{1}, \ldots, V_{k}\right)=\mathcal{V}$ & $\S 6.1$ \\
$\operatorname{disjoint}\left(\mathcal{V}, V_{1}, \ldots, V_{k}\right)$ & Vertex set pair-wise disjointness $\bigcap\left(V_{1}, \ldots, V_{k}\right)=\emptyset$ & $\S 6.1$ \\
$\operatorname{partition}\left(\mathcal{V}, V_{1}, \ldots, V_{k}\right)$ & Vertex set partition $\bigcup\left(V_{1}, \ldots, V_{k}\right)=\mathcal{V} \wedge \bigcap\left(V_{1}, \ldots, V_{k}\right)=\emptyset$ & $\S 6.1$ \\
edge $(\mathcal{G}, i, j)$ & $V A L I D$-rule: Adjacency of $(i, j)$ & $\S 6.2$ \\
$\operatorname{connected}(\mathcal{G}, i, j, \ell)$ & $V A L I D$-rule: Existence of an $\ell$-path between vertex $i$ and vertex $j$ & $\S 6.2 .1$ \\
isolated $(\mathcal{G}, i, j)$ & $V A L I D$-rule: Isolation of vertices $i$ and $j$ : There exists no path between $i$ and $j$ & $\S 6.2 .2$ \\
\hline
\end{tabular}

permutations $\pi_{\mathcal{V}}$ and $\pi_{\mathcal{E}} .{ }^{1}$ The following congruence holds in $\mathbb{Z}_{n}^{*}$ :

$$
Z \equiv \pm \underbrace{R_{\pi(i)}^{e_{i} \Pi_{k \in f_{\mathcal{V}}(i)} e_{k}}}_{\forall \text { vertices } i} \cdots \underbrace{R_{\pi(i, j)}^{e_{i} e_{j} \Pi_{k \in f_{\mathcal{E}}(i, j)} e_{k}}}_{\forall \text { edges }(i, j)} A^{e} S^{v}
$$

\subsubsection{Proof of Representation}

Establishing zero-knowledge proofs on (graph) signatures usually starts with a proof of representation: This proof shows that the prover knows all the secrets contained in the signature equation. It shows that the prover actually possesses the graph signature. To talk about further properties of the values in the signature, we need to make these values available in a commitment and thereby accessible to individual treatment. For instance, if a proof is to make statements about the vertices, we need to have commitments on the vertex identifiers and prove that the values in the commitments are equal to the values in the signature. We call this process decomposition; it creates a tree of commitments with the graph signature at its root and equality proofs connection the nodes. Whereas a proof of possession of a signature is standard, we expand on it to show how the graph signature is decomposed in commitments on its components. These decompositions implement the predicates possession, vertices, edges, and graph from Table 1 and constitute reusable building blocks for many proofs.

\section{REMARK 2 (FUll Graph DeCOMPOSITION).}

It is crucial to note that the full graph decomposition including a proof of well-formedness and pair-wise difference graph (Def. 1) is only needed when a user contributes a hidden graph. For proofs considered in this paper, the parties can trust the auditing system that it will only sign well-formed graphs, by which the infrastructure provider can limit its proof computations to the decompositionlevel required to answer the tenant's question. For instance, if the tenants asks "Were all my resources geolocated in Europe?" (a property on vertex labels), the provider computes the proof of representation possession including vertex decomposition vertices, but no edge decomposition.

\section{Commitments.}

The zero-knowledge proofs operate on committed values, which requires us to compute commitments for all values referenced in the proof. The prover computes Integer commitments on the exponents of all vertex and edge bases. First, the prover computes

\footnotetext{
${ }^{1}$ The randomization is required for multi-use unlinkability once a single graph signature is used in proofs with multiple verifiers. While the extended version specifies this in detail, we keep it her to maintain consistency.
}

commitments on all messages to allow their decomposition into components. Commit (possession $\left.\left(\mathcal{G}, \sigma, \mu_{i}\right) ; r_{i}, r_{(i, j)}\right)$ are in $\mathbb{Z}_{N}^{*}$ with uniformly chosen randomness $r_{i}, r_{(i, j)} \in\{0,1\}^{\ell}$ :

$$
\begin{aligned}
C_{i} & =R^{e_{i} \Pi_{k \in f_{\mathcal{V}}(i)} e_{k}} S^{r_{i}} \\
C_{(i, j)} & =R^{e_{i} e_{j} \Pi_{k \in f_{\mathcal{E}}(i, j)} e_{k}} S^{r_{(i, j)}}
\end{aligned}
$$

For each vertex $i$, the prover computes Commit $\left(\operatorname{vertices}(\mathcal{G}) ; \breve{r}_{i}\right)$ in $\mathbb{Z}_{N}^{*}$ on vertex attribute and identifier using uniformly-chosen randomness $\breve{r}_{i} \in\{0,1\}^{\ell}$ :

$$
\breve{C}_{i}=R^{e_{i}} S^{\breve{r}_{i}}
$$

For edges $(i, j)$, the commitments are in $\mathbb{Z}_{N}^{*}$ with uniformly chosen randomness $\breve{r}_{(i, j)}, \dot{r}_{(i, j)} \in\{0,1\}^{\ell}$ :

$$
\begin{gathered}
\text { Commit }\left(\operatorname{edges}(\mathcal{G}) ; \breve{r}_{(i, j)}, \dot{r}_{(i, j)}\right): \\
\breve{C}_{(i, j)}=R^{e_{i} e_{j}} S^{\breve{r}_{(i, j)}} \quad \text { and } \quad \dot{C}_{i}=R^{e_{i}} S^{\dot{r}_{(i, j)}} .
\end{gathered}
$$

\section{Proof of knowledge.}

We construct the proof of possession and well-formedness step by step, where it is understood the proofs will be done in one compound proof of knowledge with referential integrity between the secret exponents. Let us consider a proof fragment for vertices $i, j$ and an edge $(i, j)$ committed in a graph commitment $C$ (the same proof structure is used for CL-Signatures).

2.1 Proof of representation. We prove that commitment $C$ can be decomposed into commitments $C_{i}, C_{j}$, one for each vertex $i, j$ and one commitment $C_{(i, j)}$ for each edge $(i, j)$ :

$$
\begin{gathered}
P K\left\{\left(\mu_{i}, \mu_{j}, \mu_{(i, j)}, \rho, \rho_{i}, \rho_{j}, \rho_{(i, j)}\right): \quad(\bmod N)\right. \\
C \equiv \pm \prod_{i, j} R_{\pi(i)}^{\mu_{i}} R_{\pi(j)}^{\mu_{j}} \prod_{(i, j)} R_{\pi(i, j)}^{\mu_{(i, j)}} S^{\rho} \wedge \\
C_{i} \equiv \pm R^{\mu_{i}} S^{\rho_{i}} \wedge C_{j} \equiv \pm R^{\mu_{j}} S^{\rho_{j}} \wedge \\
\left.C_{(i, j)} \equiv \pm R^{\mu_{(i, j)}} S^{\rho_{(i, j)}}\right\} .
\end{gathered}
$$

The same proof of representation can be applied to graph signatures to prove the predicate possession:

$$
Z \equiv \pm \prod_{i, j} R_{\pi(i)}^{\mu_{i}} R_{\pi(j)}^{\mu_{j}} \prod_{(i, j)} R_{\pi(i, j)}^{\mu_{(i, j)}} A^{\prime \varepsilon^{\prime}} S^{\rho^{\prime}}
$$

2.2 Vertex composition. Second, we need to show properties of the vertex composition that the encoding for each vertex $i$ contains exactly one vertex identifier $e_{i} \in \Xi_{\mathcal{V}}$ and zero or multiple label representatives $e_{k} \in \Xi_{\mathcal{L}}$. We show this structure with help of 
the commitments $\breve{C}_{i}$ and set membership and prime-encoding OR proofs. This proof is executed for all vertices.

$$
\begin{aligned}
P K\left\{\left(\forall i: \varepsilon_{i}, \breve{\rho}_{i}, \gamma_{i}, \rho_{i}^{\prime}\right):(\bmod N)\right. \\
\breve{C}_{i} \equiv \pm R^{\varepsilon_{i}} S^{\breve{\rho_{i}}} \wedge C_{i} \equiv \pm \breve{C}_{i}^{\gamma_{i}} S^{\rho_{i}^{\prime}} \wedge \\
\left.\gamma_{i}\left[C_{i}\right] \subseteq \Xi_{\mathcal{L}} \wedge \varepsilon_{i}\left[\breve{C}_{i}\right] \in \Xi \mathcal{V}\right\} .
\end{aligned}
$$

Clause 4 establishes the predicate vertices $\left(\mathcal{G}, \mu_{i}, \varepsilon_{i}, \gamma_{i}\right)$, where its second sub-clause links to the possession commitments.

2.3 Edge composition. Third, we prove the structure of each edge $(i, j)$ over the commitments $C_{(i, j)}$, showing that each commitment contains exactly two vertex identifiers $e_{i}, e_{j} \in \Xi_{\mathcal{V}}$ as well as zero or more label representative $e_{k} \in \Xi_{\mathcal{L}}$ :

$$
\begin{gathered}
P K\left\{\left(\dot{\varepsilon}_{i}, \dot{\varepsilon}_{j}, \rho_{(i, j)}, \gamma_{(i, j)}, \rho_{(i, j)}^{\prime}\right):(\bmod N)\right. \\
\breve{C}_{(i, j)} \equiv \pm \dot{C}_{i}^{\dot{\varepsilon}_{j}} S^{\rho_{(i, j)}} \wedge \dot{C}_{i} \equiv \pm R_{i}^{\dot{\varepsilon}_{i}} S^{\dot{\rho}_{i}} \\
C_{(i, j)} \equiv \pm \breve{C}_{(i, j)}^{\gamma_{(i, j)}} S^{\rho^{\prime}{ }_{(i, j)}} \wedge \\
\left.\left.\gamma_{(i, j)} \subseteq \Xi_{\mathcal{L}} \wedge \dot{\varepsilon}_{j}\left[\breve{C}_{(i, j)}\right] \in \Xi_{\mathcal{V}}\right\}\right\} .
\end{gathered}
$$

Clauses 6 and 7 realize the predicate edges $\left(\mathcal{G}, \varepsilon_{i}, \varepsilon_{j}, \gamma_{(i, j)}\right)$, for which Clause 7 binds the edges commitments to the possession commitments. Clauses 5 and 8 establish that identifiers and labels are valid.

2.4 Pair-wise difference. We give the proof of pair-wise difference of vertices for completeness; it is not required in the application scenario in which we trust the audition system to only sign well-formed graphs. We show that the vertex representatives are pair-wise co-prime over the commitments $\breve{C}_{i}$ and $\breve{C}_{j}$.

$$
\begin{aligned}
& P K\left\{\left(\forall i, j: \alpha_{i, j}, \beta_{i, j}, \rho_{i, j}\right):\right. \\
&\left.R \equiv \pm \breve{C}_{i}^{\alpha_{i, j}} \breve{C}_{j}^{\beta_{i, j}} S^{\rho_{i, j}}(\bmod N)\right\} .
\end{aligned}
$$

THEOREM 5.1 (PROOF OF WELL-FORMEDNESS). The compound proof of knowledge establishes the well-formedness of an encoded graph according to Def. 1.

[Proof in [26]]

\section{PROOFS OF GRAPH PROPERTIES}

Having established encoding and foundational bootstrapping cycle of proof of representation and issuing, we continue to establish a library of graph proof predicates. First, we explore with proofs over vertex and edge sets, including coverage and pair-wise disjointness, which are the basis of partition proofs. Second, we discuss different proofs over presence and absence of labels. Third, we establish results on connectivity and isolation in undirected graphs.

\subsection{Sets as Cumulative Products}

The idea to reach a compact set representation is: given that the elements of the set are all primes, we can safely multiply all set members without losing access to individual values. Hence, we represent a set of vertices $V \subseteq \mathcal{V}$ with $\ell$ elements by the cumulative product of its vertex identifiers:

$$
E_{V}=\Pi_{i \in V} e_{i} .
$$

The normal set representation only includes the vertex identifiers of the vertex set, the extended set representation also all associated vertex labels. An edge set is represented at the cumulative product of all the vertex identifiers of the edges involved. We will use proofs over cumulative products repeatedly and establish a generic interface for those. In the following, we rename the vertex identifier indices to range over $1, \ldots, \ell$, without loss of generality.
1. Commitments. The prover commits to the vertex set representation as the cumulative product $E_{V}$. To prepare the proof of representation the prover establishes intermediate commitments Commit $\left(\operatorname{set}(\mathcal{V}, V) ; \breve{r}_{1}, \ldots, \breve{r}_{\ell}\right)$ in $\mathbb{Z}_{N}^{*}$ on partial products using uniformly chosen randomness $\breve{r}_{1}, \ldots, \breve{r}_{\ell}$ :

$$
\breve{C}_{V, 1}=R^{e_{1}} S^{\breve{r}_{1}}, \ldots, \breve{C}_{V, \ell}=R^{\Pi_{1}^{\ell} e_{i}} S^{\breve{r}_{\ell}} .
$$

2. Proof of Representation. To establish a proof of representation of a set $\operatorname{set}(\mathcal{V}, V)$ that a cumulative product $E_{V}$ is composed of the identifiers of certified vertices, the prover engages with the verifier in the following proof of knowledge over the cumulative product:

$$
\begin{aligned}
& P K\left\{\left(\left(\forall i: \mu_{i}, \varepsilon_{i}\right), \breve{\rho}_{1}, \ldots, \breve{\rho}_{\ell}\right): \quad(\bmod N)\right. \\
& \operatorname{possession}\left(\mathcal{G}, \sigma, \mu_{i}\right) \wedge \operatorname{vertices}\left(\mathcal{G}, \mu_{i}, \varepsilon_{i}\right) \\
& \breve{C}_{V, 1} \equiv \pm R^{\varepsilon_{1}} S^{\breve{\rho}_{1}} \wedge \\
& \breve{C}_{V, 2} \equiv \pm \breve{C}_{V, 1}^{\varepsilon_{2}} S^{\breve{\rho}_{2}} \wedge \ldots \\
& \breve{C}_{V, \ell} \equiv \pm \breve{C}_{V, \ell-1}^{\varepsilon_{\ell}} S^{\rho_{\ell}}
\end{aligned}
$$

REMARK 3 (EDGE SETS).

Edge sets can be represented as products of their vertex identifiers, as well. This is a degenerate representation as it does not maintain the edge structure, but only the vertices present, however as we shall see in \$6.2.1 it serves its purpose for edge partitions.

\subsubsection{Coverage}

We want to find out whether a given set of vertex sub-sets completely covers the entire graph. The predicate cover establishes $\bigcup\left\{V_{1}, V_{2}, \ldots, V_{k}\right\} \supseteq \mathcal{V}$. As the set is represented as product of vertex identifiers, this equivalent to the product of all vertex identifiers of the graph $\chi_{\mathcal{V}}$ dividing the product representation of the sets:

$$
\chi \mathcal{V} \mid \prod_{i=1}^{k} E_{V_{i}} \Leftrightarrow \exists a: a \chi \mathcal{V}=\prod_{i=1}^{k} E_{V_{i}}
$$

where $E_{V_{i}}=\prod_{j \in V_{i}} e_{j}$. The product representation of $\Xi_{\mathcal{V}}$ is signed with $C_{\mathcal{V}}$ as part of the issuing. Thus, given proofs for $\operatorname{set}\left(\mathcal{G}, V_{i}\right)$, we compute $\operatorname{set}\left(\mathcal{G},\left\{V_{1}, \ldots, V_{k}\right\}\right)$, which results in a commitment on the cumulative product of all sets:

$$
C_{\bar{V}}=R \prod_{i=1}^{k} E_{V_{i}} S^{\bar{r}} .
$$

To complete the proof of coverage, we prove that the cumulative product over all subset in the commitment $C_{\bar{V}}$ divides the product of all vertex identifiers $C_{\mathcal{V}}$.

$$
\begin{aligned}
P K & \{(\alpha, \rho): \\
& \operatorname{set}\left(\mathcal{G}, V_{1}\right) \wedge \cdots \wedge \operatorname{set}\left(\mathcal{G}, V_{k}\right) \wedge \\
& \operatorname{set}\left(\mathcal{G},\left\{V_{1}, \ldots, V_{k}\right\}\right) \wedge \\
& C_{\bar{V}} \equiv \pm C_{\mathcal{V}}^{\alpha} S^{\rho}(\bmod N)
\end{aligned}
$$

\subsubsection{Pair-wise Disjointness}

The predicate disjoint establishes that vertex sets have no joint vertex, $\bigcap\left\{V_{1}, V_{2}, \ldots, V_{k}\right\}=\emptyset$. Recall that the sets are products of primes. Hence, we can use the fact that two vertex sets $V_{i} \subseteq V$ and $V_{j} \subseteq \mathcal{V}$ are pair-wise disjoint if their product representations are coprime:

$$
V_{i} \cap V_{j}=\emptyset \quad \Leftrightarrow \quad \operatorname{gcd}\left(E_{V_{i}}, E_{V_{j}}\right)=1 .
$$


If two sets share a vertex, i.e., a prime factor, this equation cannot be proven.

Based on given commitments $C_{V_{i}}$ and predicates $\operatorname{set}\left(\mathcal{G}, V_{i}\right)$ and $\operatorname{set}\left(\mathcal{G}, V_{j}\right)$, we establish coprimality and thereby disjointness with Bézout's Identity,

$$
\operatorname{gcd}\left(E_{V_{i}}, E_{V_{j}}\right)=1 \quad \Leftrightarrow \quad a E_{V_{i}}+b E_{V_{j}}=1,
$$

resulting in the following proof:

$$
\begin{gathered}
P K\left\{\left(\forall i, j: \alpha_{i, j}, \beta_{i, j}, \rho_{i, j}\right): \quad(\bmod N)\right. \\
\operatorname{set}\left(\mathcal{G}, V_{1}\right) \wedge \cdots \wedge \operatorname{set}\left(\mathcal{G}, V_{k}\right) \wedge \\
\forall i, j: R \equiv \pm C_{V_{i}}^{\alpha_{i, j}} C_{V_{j}}^{\beta_{i, j}} S^{\rho_{i, j}}
\end{gathered}
$$

\subsubsection{Partition}

In a partition proof we seek to establish that a several sets of vertices cover the entire graph and that they are all pair-wise disjoint. Hence, we combine both proofs above on coverage and pair-wise disjointness operating on commitments for the predicate $\operatorname{set}\left(\mathcal{G}, V_{i}\right)$.

\subsection{Statements over Edges}

We now turn our attention to edges and connectivity, where we start with existence of a single before we proceed with connectivity and isolation.

Here we seek to prove that there exists an edge between two secret vertices, that is, the edge predicate. Thus, we need to show that there exists an edge base with an exponent $E$ which encodes the edge $(i, j)$. This, in turn, means that the product of the edge's vertex identifiers divides the exponent.

$$
\left(e_{i} e_{j}\right) \mid E \Leftrightarrow \exists a: a\left(e_{i} e_{j}\right)=E .
$$

This is realized by the prover engaging with the verifier in the following proof of knowledge:

$$
\begin{aligned}
& P K\left\{\left(\left(\forall i: \mu_{i}\right), \mu^{\prime}, \rho^{\prime}\right):\right. \\
& \quad \operatorname{possession}\left(\mathcal{G}, \mu_{i}\right) \wedge \\
& \left.\quad C_{(i, j)} \equiv \pm\left(R^{e_{i} e_{j}}\right)^{\mu^{\prime}} S^{\rho^{\prime}}(\bmod N)\right\} .
\end{aligned}
$$

\subsubsection{Connectivity}

A major area of interest for hidden-graph proofs is connectivity: How can we show that different vertices of a hidden graph are connected by a chain of $\ell$ edges? The predicate connected $(\mathcal{G}, i, j, \ell)$ means that there exists a sequence of at most $\ell$ edges, such that the end vertex of one edge is the start vertex of the next.

EXAMPLE 1.

Let us consider the following chain of connected edges:

$$
\left(e_{i} \cdot e_{1}\right),\left(e_{1} \cdot e_{2}\right),\left(e_{2} \cdot e_{3}\right),\left(e_{3} \cdot e_{j}\right)
$$

We observe that two vertices are connected if and only if there exists a sequence of edge products, such that their the edges match in the joint vertex identifier. By that, we have for instance for the first pair of edges:

$$
e_{1}\left|\left(e_{i} \cdot e_{1}\right) \wedge e_{1}\right|\left(e_{1} \cdot e_{2}\right)
$$

The idea here is to prove connectivity by showing that the vertex identifier of the connection point between two adjacent edges divides both edge representations. We will chain these divisibility proofs.

Recall that the predicate edges $\left(\mathcal{G}, \varepsilon_{i}, \varepsilon_{j}, \gamma_{(i, j)}\right)$ from cf. $\$ 5.3 .1$ computes the following commitments in $\mathbb{Z}_{N}^{*}$ :

$$
\breve{C}_{(i, j)}=R^{e_{i} e_{j}} S^{\breve{r}_{(i, j)}} \quad \text { and } \quad \dot{C}_{i}=R^{e_{i}} S^{\dot{r}_{(i, j)}}
$$

for each edge $(i, j)$, allowing us to compose statements over adjacent edges via $\breve{C}_{(i, j)}$ and the subsequent $\dot{C}_{j}$. We show that a joint factor $\varepsilon$ divides adjacent edges $(i, j)$ and $(j, k)$ with the following proof of knowledge:

$$
\begin{aligned}
P K\left\{\left(\varepsilon, \rho, \rho^{\prime}\right):\right. & (\bmod N) \\
& \left.\breve{C}_{(i, j)} \equiv \pm \dot{C}_{i}^{\varepsilon} R^{\rho} \wedge \dot{C}_{j}=R^{\varepsilon} S^{\rho^{\prime}}\right\} .
\end{aligned}
$$

\subsubsection{Isolation}

Proving isolation of sub-graphs in zero-knowledge efficiently is a daunting task as the prover could "forget" an edge, which normally forces the proof to iterate over all edges (quadratic complexity). We can do better: We can partition the graph into two edge sets, each containing one vertex of the isolation claim. If there is any connection between these two sets, then there must be an edge from one set two the other, which means that they have a joint factor.

For vertices $i$ and $j$ isolated $(i, j)$ means that there exists no connected path between both vertices $i$ and $j$. For undirected graphs, isolation means that the vertices $i$ and $j$ are in separate sub-graphs. Two vertices $i$ and $j$ are isolated, if there exists a bi-partition of the edge set $V^{\prime} \cup V^{\prime \prime}=\mathcal{E} \wedge V^{\prime} \cap V^{\prime \prime}=\emptyset$, such that without loss of generality $i \in V^{\prime}$ and $j \in V^{\prime \prime}$. Recall that $\$ 6.1$ represents an edge set in a degenerate form, as the product of the edges' vertex identifiers $E^{\prime}$ and $E^{\prime \prime}$. We obtain commitments and proofs for the predicates $\operatorname{set}\left(\mathcal{G}, V^{\prime}\right)$ and $\operatorname{set}\left(\mathcal{G}, V^{\prime \prime}\right)$ which give us two commitments in $\mathbb{Z}_{N}^{*}$ :

$$
\breve{C}_{E^{\prime}}=R^{E^{\prime}} S^{\breve{r}^{\prime}} \quad \text { and } \quad \breve{C}_{E^{\prime \prime}}=R^{E^{\prime \prime}} S^{\breve{r}^{\prime \prime}} .
$$

We can derive coverage already from the fact that all commitments of the predicate edges are used to establish the cumulative products for both edge sets. The disjointness of the edge-set bi-partition gives the isolation result, which we prove by showing that both products are coprime:

$$
\operatorname{gcd}\left(E^{\prime}, E^{\prime \prime}\right)=1 \quad \Leftrightarrow \quad \exists a, b: a E^{\prime}+b E^{\prime \prime}=1 .
$$

The complete the proof of the predicate isolated, the prover and the verifier engage in the following proof of knowledge:

$$
\begin{gathered}
P K\{(\alpha, \beta, \rho): \quad(\bmod N) \\
\operatorname{set}\left(\mathcal{G}, V^{\prime}\right) \wedge \operatorname{set}\left(\mathcal{G}, V^{\prime \prime}\right) \wedge \\
R \equiv \pm \breve{C}_{E^{\prime}}^{\alpha} \breve{C}_{E^{\prime \prime}}^{\beta} S^{\rho}
\end{gathered}
$$

REMARK 4 (ISOLATION ON VLAN IDS).

The isolation predicate constructed in this section argues over the edges only. In actual topologies, such as infrastructure clouds, it is however the case that graph labels are important to decide upon connectivity and isolation. This holds in particular for the VLAN IDs of virtualized infrastructures, which allow communication if components have matching VLAN IDs. The isolation predicate can be easily extended to show pair-wise disjointness for labels as well.

\section{PERFORMANCE EVALUATION}

\subsection{Asymptotic Evaluation}

We display the efficiency analysis for the proof predicates in Table 2, where each row shows the overhead over the basis predicate stated in the first column. We measure computational complexity in multi-base and modular exponentiations. The communication complexity is dominated by the transmitted group elements from $\mathbb{Z}_{N}^{*}$, which is equal to the number of multi-base exponentiations (one for each Integer and Schnorr proof commitment). In the scenario we consider in this paper, the verifier trusts the issuer to only certify 
well-formed graphs. As we do not need to prove well-formedness of a hidden graph, we have linear complexity for all proofs.

The modular exponentiations for message bases $R_{i}$ have small exponents of size $\ell_{\mathcal{M}} \ll \ell_{N}$, where the parameter $\ell_{\mathcal{M}}$ can be chosen similarly small as in Direct Anonymous Attestation [8]. Implementing the proofs of representation as multi-base exponentiations will reduce the number of multiplications significantly and thereby offer a significant speed-up. In addition, the $\Sigma$-proofs employed in this work benefit from batch-proof techniques [32]. The system allows signature proofs of knowledge with the Fiat-Shamir heuristic [20], which can be computed offline.

We note that the public key size (that is the certification of vertex and label identifiers) is linear in the maximal number of vertices and label types, a case in point to partition the infrastructure representation and cover it with multiple (hierarchical) graph signatures.

\subsection{Experimental Evaluation}

Figure 2 contains an experimental performance analysis, based on the following parameters: The issuer has established the setup with Quadratic Residues $\mathrm{QR}_{N}$ under a special RSA modulus $N$ as specified by the Identity Mixer Library, where the modulus length $\ell_{n}=2048$ bits and system parameters $\left(\ell_{v}\right.$, etc.) are chosen exactly as prescribed in the library setup [27]. Bases for vertex and edge encoding are computed according to the library setup. The performance analysis is executed on 64-bit Java JDK 1.7.13 on a Windows 7 SP 1 Thinkpad X220 Tablet, on Intel CPU i5-2520 with $2.5 \mathrm{GHz}, 8 \mathrm{~GB}$ RAM, where all computations are performed on a single processor core only. This is a very conservative estimate as an infrastructure provider can use multiple cores and parallelize the computations on all levels. The performance analysis uses the math utility functions of the Identity Mixer Library for the computation of randomness and exponentiations, that is, its MultiExp facility.

The experiments performed on a prototypical implementation of computations required for the graph signature scheme, that is, on representative computations of commitments and a proof of knowledge thereof. For a commitment, we would have a structure $C:=R_{0}^{m_{0}} \cdots R_{\ell}^{m_{\ell}} S^{v} \bmod N$, where the exponents are uniform random bit-strings of the prescribed length and number (as in the actual Schnorr proof witnesses). The simulation uses random graphs with specified number of vertices $n$ and a derived number of edges $m:=2 n$ as major independent variable (on the $x$-axis), the dependent variable is computation time in milliseconds (in logscale on the $y$-axis). Again, these computations give a conservative estimate of the expected computation time: issuing and proofs that disclose attributes operate on smaller exponents, by which they are more efficient. Any implementation on actual graphs, with multiple cores or parallelization strategies will perform much better.

\section{Discussion.}

If we consider the computations done by the issuer, we see that the issuer can sign large graphs with 10,000 vertices and 20,000 edges (a realistic size for an in-house cloud) in 532 milliseconds, if the issuer uses his knowledge of the discrete-logarithms between generator and bases for optimization ${ }^{2}$. This means the issuer can sign large graphs rapidly. The user's proof of representation of a signature with 10,000 vertices and 20,000 edges, in turn, can be performed in a time of 238 seconds, $\approx 4$ minutes, which is feasible again. We believe that the usual use case will be that the size of the user-contributed subgraph is very small compared to the size

\footnotetext{
${ }^{2}$ An implementation using the standard CL-issuing without this optimization will use 476 seconds, $\approx 8$ minutes
}

of the issuer-contributed subgraph. Such use cases can thereby be implemented efficiently.

EXAMPLE 2 (VIRTUALIZED INFRASTRUCTURE). Let us consider the scenario of the system model of $\$ 2$ again. The auditing system certifies the virtualized infrastructure after a security analysis and issues a graph signature to the infrastructure provider. The auditor gets privileged access to the infrastructure, hence, no user-generated proof of well-formedness of a committed graph is needed. The auditing system can issue signatures with a frequency in the order of seconds. Upon tenant request with a time index $t$, the infrastructure provider looks up the appropriate graph signature for time $t$ to prove to tenants in zero-knowledge that security properties, such as isolation from competitors, are fulfilled. The provider fixes a time of infrastructure state and corresponding signature, and computes the proof of knowledge disclosing only the properties in question, in the order of minutes. We expect the tenants will only query the provider sporadically for proofs over the graph signatures.

REMARK 5 (SCALABILITY).

First, we note that the core algorithms have linear complexity in number of vertices and edges, with a low slope constant. Second, we observe that the decomposition is highly parallelizable: All information (message blocks and random exponents) is known a priori and there are no state inter-dependencies between commitment computations or Schnorr proofs: The commitments can be computed in parallel and once they are computed the Schnorr proofs can be computed in parallel, as well. This requires computing all the witnesses first, then synchronize the computation results to compute the challenge with the Fiat-Shamir heuristic, to finally compute all the responses in parallel again. Thus, we believe graph signatures to be scalable.

\section{RELATED WORK}

\section{Structural and Topological Cloud Assurance.}

Whereas past work was often on hardening hypervisors and preventing isolation breaches in the systems themselves, there are multiple lines of work in the (structural) security assurance of virtualized infrastructures assuming that the hypervisors and management hosts are correct, yet might be misconfigured. Zhai et al. [38] proposed a structural reliability auditor, which discovers dependencies between cloud components. This was later extended by Xiao et al. [37] to achieve this privately in secure multi-party computation (SMPC). Their work argues over the dependency graph of cloud components, a data structure which can be directly implemented in the graph signature scheme presented here. The goal of our works is different, however: Whereas they allow multiple parties (e.g., providers) to compute jointly whether there are hidden dependencies without disclosing secret information, our work proves properties of audited dependency graph, e.g., absence of a single-point-of-failure or isolation from a particular dependency, in zero-knowledge.

SAVE and subsequent works of Bleikertz et al. [4, 3] pursue security analysis of virtualized infrastructures based on a graph representation. Their work employs discovery probes or sensors for virtualized infrastructure change events to maintain a graph representation in sync with the actual topology of the infrastructure. Their work first focused on information flow analysis by graph coloring, yet has been extended subsequently with general purpose model checkers, verifying the graph model against a high-level security policy in abstract language, either the set rewriting dialect 
Table 2: Efficiency of proofs of predicates in multi-base and modular exponentiations (MultiExps and ModExps). For a simple graph holds $m \leq \frac{n(n-1)}{2}$. Note that the constant $k$ is the number of sets considered, e.g., $k=2$ for a bipartition. In practice holds $k \ll \ell \leq n$ and $O(k \ell)^{2}=O(n)$, hence, all proofs are linear in $n$ or $m$.

\begin{tabular}{llcccc}
\hline Predicate & Basis & $\begin{array}{c}\text { Commitments } \\
\text { MultiExps }\end{array}$ & M & ModExps & $O$ \\
\hline possession $\left(\mathcal{G}, \sigma, \mu_{i}\right)$ & & $n+m$ & $2 n+2 m+1$ & $5 n+5 m+2$ & $O(n+m)$ \\
$\operatorname{vertices}(\mathcal{G})$ & possession & $n$ & $3 n$ & $6 n$ & $O(n)$ \\
$\operatorname{edges}(\mathcal{G})$ & possession & $2 m$ & $4 m$ & $8 m$ & $O(m)$ \\
$\operatorname{set}(\mathcal{V}, V)$, with $\ell=|V|$ & vertices & $\ell$ & $2 \ell$ & $4 \ell$ & $O(\ell)=O(n)$ \\
$\operatorname{cover}\left(\mathcal{V}, V_{1}, \ldots, V_{k}\right)$ & vertices & $k(\ell+1)$ & $2 k(\ell+1)+1$ & $4 k(\ell+1)+2$ & $O(k \ell)$ \\
$\operatorname{disjoint}\left(\mathcal{V}, V_{1}, \ldots, V_{k}\right)$ & vertices & $k \ell$ & $k^{2}+2 k \ell$ & $3 k^{2}+4 k \ell$ & $O\left(k^{2}+k \ell\right)$ \\
$\operatorname{partition}\left(\mathcal{V}, V_{1}, \ldots, V_{k}\right)$ & vertices & $k(\ell+1)$ & $k^{2}+2 k(\ell+1)$ & $3 k^{2}+4 k(\ell+1)+2$ & $O\left(k^{2}+k \ell\right)$ \\
$\operatorname{edge}(\mathcal{G}, i, j)$ & possession & 0 & 1 & 2 & $O(1)$ \\
$\operatorname{connected}(\mathcal{G}, i, j, \ell)$ & edges & 0 & $2 \ell$ & $4 \ell$ & $O(\ell)=O(m)$ \\
isolated $(\mathcal{G}, i, j)$ & edges & $m$ & $2 m+1$ & $4 m+3$ & $O(m)$ \\
\hline
\end{tabular}

VALID [2] or graph rewriting languages. The most recent advances in that line of work include a fast differential analysis on change events. Their graph representation and information flow overlay can serve as basis for this work's auditing system and issuing of graph signatures. At the same time, the tenant can query for zeroknowledge proofs based on the security policies employed. By that, our work could extend theirs by a projection of trust: Whereas Bleikertz et al. have the infrastructure provider specify the security policy for the analysis, run the analysis tool, and have the tenant trust that all this was done correctly, the provider can now delegate running the tool to a trusted auditor and satisfy the tenants own security policies in zero-knowledge.

TPM-protected host-based monitoring of virtualized infrastructures offers an alternative to structural security assurance. Cloud Verifier [34], for example, allows a remote tenant to specify integrity criteria for which Cloud Verifier will monitor a node server. This approach employs an integrity verification proxy as well as TPM support for attestation. The monitored policies include VMinfos, the network and host security policies and the host memory, all of which are limited to host properties. Our approach of graph signatures is complementary to host-based auditing, as it allows for proofs over the inter-connectivity of components beyond the host's boundaries. For instance, a host's assurance of a correct VLAN ID configuration is not sufficient for VLAN-based network isolation, as there could be a VLAN ID collision anywhere in the network. Similarly, a host's assurance that it is connected to the correct remote storage partition does not guarantee that there is no other component connected to that partition.

\section{Zero-Knowledge Proofs and Signatures on Graphs.}

Zero-knowledge proofs on graphs and their properties is a classic area of research and have been instrumental in showing that there exist zero-knowledge proof systems for all NP languages, e.g., [22, 5] Both proofs use a metaphor of locked boxes to construct knowngraph proofs of Graph 3-Colorability (G3C) or Directed Hamiltonian cycles (DHC). The constructions focus on zero-knowledge proofs of knowledge and do not cater for a level of indirection through a signature scheme or proofs of knowledge on graph properties in a hidden-graph setting.

A related notion to full graph signatures is transitive or homomorphic signature schemes, such as $[29,28,1]$. They are concerned with the transitive closure of signatures on graph elements, such that from signatures from edges $(i, j)$ and $(j, k)$ everybody can derive a valid signature on the edge $(i, k)$. These signature schemes have the advantage that one can produce a signature of the transitive path over multiple edges. Therefore, they allow showing signatures equivalent to the connected predicate without disclosing the number of edges on the path and without overhead because of path length. The constructions are not meant to be on committed graphs and consider the signatures as public information. They have limited support for labels and do not have provisions for proofs of isolation as signatures could be withheld.

\section{Authenticated and Verifiable Graph Computations.}

Authenticated data structures for graph connectivity have been thoroughly investigated by Goodrich et al. [25], whose approach is restricted to hash-based authentication and does not yield zeroknowledge properties. Subsequent work [24] has been applied to Web-content searching and proposed an authenticated web crawler, whose problem statement also exhibits the highly dynamic nature of the authenticated data structure. The authentication is based on a root-signed Merkle tree, which prevents a zero-knowledge access to the underlying data structure as pursued in this work. The intersection proofs based on the certification of succinct relations presents an interesting avenue for future graph proofs.

One can realize graph proofs with Verifiable Computation (VC), in which a client outsources a computation to an untrusted worker and is subsequently enabled to verify the correctness of the computation result. The recent Pinocchio scheme by Parno et al. [30] employs quadratic programs to achieve a highly efficient verification and constant-size proofs of computation. Whereas the proofs of computation can be made statistically zero-knowledge at low cost, the zero-knowledge certification of the auxiliary worker input (in our case the topology graph) is not considered. However, VC may provide an alternative to compiling $\Sigma$-proofs on the graph signature. In parallel to this work, Zhang et al. [39], have proposed ALITHEIA, a verifiable graph processing framework and offered a comprehensive comparison of Verifiable Computing results on graphs. Zhang et al. state that the verifiable execution of BFS on Pinocchio suffered from the need to be expressed as a circuit and the involved quadratic blow-up, which resulted in an implementation scaling up only to 50 vertices. The $15 \mathrm{~GB}-\mathrm{RAM}$ experiment machine was running out of memory when compiling the certifying algorithm code on graphs with more than 10,000 vertices. Thus, whereas the verification time offered by the ALITHEIA graph VC only grows sub-linearly and can be considerably faster than the compiled Schnorr proofs we use in this approach, this comes at a high storage cost for the server. 


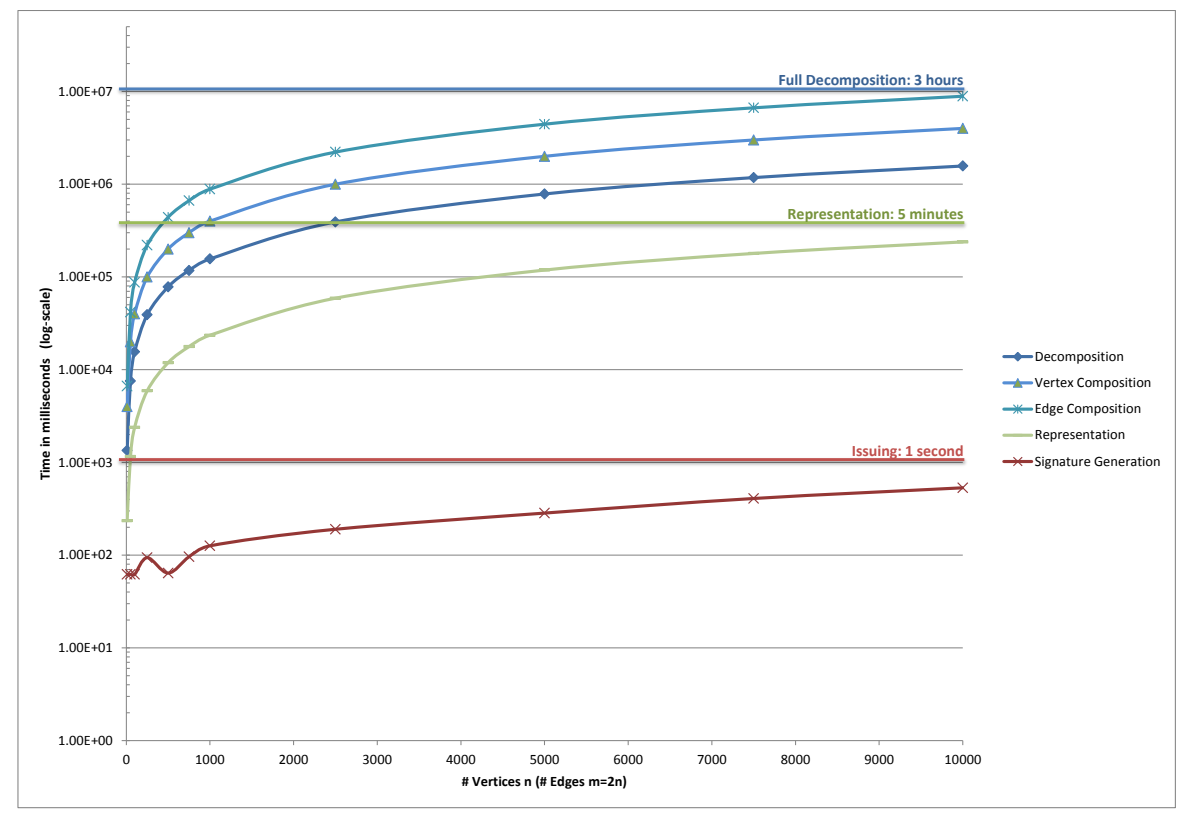

Figure 2: Experimental performance analysis with a secure modulus length of 2048 bits, in the worst case of a non-parallelized computation on a single processor core. $x$-axis contains the number of vertices $n$ and the $y$-axis a log-scale of computation time in milliseconds. Blue colors denote provider computations to prove properties of a committed graph, where the green line shows a proof of representation of a graph signature. Red colors denote auditing system/issuer computations to sign the graph.

\section{CONCLUSION AND FUTURE WORK}

We have introduced a signature scheme on committed graphs together with a framework of proof predicates on sets, connectivity and isolation. The scheme covers undirected, unlabeled and labeled graphs and enables honest-verifier zero-knowledge proofs of knowledge over graph properties, while keeping the graph itself confidential. It constitutes a building block to overcome the requirement gap between the confidentiality requirements of a provider and the integrity requirements of a tenant.

The signature scheme and its proofs are created in a special RSA setting; their security is based on the Strong RSA assumption. The signature scheme is based on the Camenisch-Lysyanskaya (CL) signature scheme [11] and its existential unforgeability directly derived from that. The proofs can be transformed to signature proofs of knowledge with the Fiat-Shamir [20] heuristic. The constructions for the proof predicates are efficient and practical; their performance can be vastly improved with parallelization.

As future work, we see great potential in linking the graph signatures to Direct Anonymous Attestation (DAA) [8]. This allows the combination of attestation results for system components (e.g., physical and virtual machines) with statements over the system topology. Furthermore, we believe that a systematic parallelization and a differential approach will allow graph signatures and corresponding proof systems perform well, by which we will pursue a cloud implementation of the signing and decomposition proofs.

\section{Acknowledgments}

The author's research on graph signatures currently supported by the EU FP7 FutureID project (http: / / futureid.eu) under GA $n^{o} 318424$. We are grateful for the initial discussions with Jens Groth and Jan Camenisch. This work has benefited from the reviews and insightful comments of the anonymous reviewers of EUROCRYPT 2014, the IEEE Security and Privacy Symposium 2014, ACM CCS 2014 and ACM CCSW 2014.

\section{REFERENCES}

[1] Bellare, M., and Neven, G. Transitive signatures based on factoring and rsa. In Advances in Cryptology ASIACRYPT 2002. Springer, 2002, pp. 397-414.

[2] Bleikertz, S., AND Gross, T. A Virtualization Assurance Language for Isolation and Deployment. In IEEE International Symposium on Policies for Distributed Systems and Networks (POLICY'11) (Jun 2011), IEEE.

[3] Bleikertz, S., Gross, T., AND Mödersheim, S. Automated Verification of Virtualized Infrastructures. In ACM Cloud Computing Security Workshop (CCSW'11) (Oct 2011), ACM.

[4] Bleikertz, S., Gross, T., Schunter, M., And ERIKSSON, K. Automated Information Flow Analysis of Virtualized Infrastructures. In 16th European Symposium on Research in Computer Security (ESORICS'11) (Sep 2011), Springer.

[5] BLUM, M. How to prove a theorem so no one else can claim it. In Proceedings of the International Congress of Mathematicians (1986), vol. 1, p. 2.

[6] Boudot, F. Efficient proofs that a committed number lies in an interval. In Advances in Cryptology - EUROCRYPT 2000 (2000), B. Preneel, Ed., vol. 1807 of Lecture Notes in Computer Science, Springer Verlag, pp. 431-444.

[7] BRANDS, S. Rapid demonstration of linear relations connected by boolean operators. In Advances in Cryptology - EUROCRYPT '97 (1997), W. Fumy, Ed., vol. 1233 of Lecture Notes in Computer Science, Springer Verlag, pp. 318-333.

[8] Brickell, E., Camenisch, J., And Chen, L. Direct anonymous attestation. In Proc. 11th ACM Conference on Computer and Communications Security (2004), acm press, pp. 225-234. 
[9] Camenisch, J., ChaAbouni, R., And Shelat, A. Efficient protocols for set membership and range proofs. In Advances in Cryptology-ASIACRYPT 2008 (2008), Springer, pp. 234-252.

[10] CAMEnisch, J., AND GRoss, T. Efficient attributes for anonymous credentials. ACM Transactions on Information and System Security (TISSEC) 15, 1 (2012), 4.

[11] CAmenisch, J., AND Lysyanskaya, A. A signature scheme with efficient protocols. In Security in Communication Networks SCN 2002 (2003), vol. 2576 of LNCS, Springer Verlag, pp. 268-289.

[12] Camenisch, J., And Michels, M. Proving in zero-knowledge that a number $n$ is the product of two safe primes. In Advances in Cryptology - EUROCRYPT '99 (1999), J. Stern, Ed., vol. 1592 of Lecture Notes in Computer Science, Springer Verlag, pp. 107-122.

[13] CAmenisch, J., AND StAdLER, M. Efficient group signature schemes for large groups. In Advances in Cryptology - CRYPTO '97 (1997), B. Kaliski, Ed., vol. 1296 of Lecture Notes in Computer Science, Springer Verlag, pp. 410-424.

[14] Chan, A., Frankel, Y., And Tsiounis, Y. Easy come easy go divisible cash. In Advances in Cryptology EUROCRYPT '98 (1998), K. Nyberg, Ed., vol. 1403 of Lecture Notes in Computer Science, Springer Verlag, pp. 561-575.

[15] Chaum, D., And Pedersen, T. P. Wallet databases with observers. In Advances in Cryptology - CRYPTO '92 (1993), E. F. Brickell, Ed., vol. 740 of Lecture Notes in Computer Science, Springer-Verlag, pp. 89-105.

[16] Cramer, R., DAMgÅRD, I., AND Schoenmakers, B. Proofs of partial knowledge and simplified design of witness hiding protocols. In Advances in Cryptology - CRYPTO '94 (1994), Y. G. Desmedt, Ed., vol. 839 of LNCS, Springer Verlag, pp. 174-187.

[17] CSA. The Notorious Nine: Cloud Computing Top Threats in 2013. Tech. rep., Cloud Security Alliance (CSA), Feb. 2013.

[18] DAMGÅRD, I., AND FUJISAKI, E. An integer commitment scheme based on groups with hidden order. In Advances in Cryptology - ASIACRYPT 2002 (2002), vol. 2501 of Lecture Notes in Computer Science, Springer.

[19] ENISA. Cloud computing: Benefits, risks and recommendations for information security, rev. b. Tech. rep., European Network and Information Security Agency (ENISA), Dec. 2012.

[20] FiAT, A., AND Shamir, A. How to prove yourself: Practical solutions to identification and signature problems. In Advances in Cryptology - CRYPTO '86 (1987), A. M. Odlyzko, Ed., vol. 263 of Lecture Notes in Computer Science, Springer Verlag, pp. 186-194.

[21] FuJisaki, E., AND OKAMOTo, T. Statistical zero knowledge protocols to prove modular polynomial relations. In Advances in Cryptology - CRYPTO '97 (1997), B. Kaliski, Ed., vol. 1294 of Lecture Notes in Computer Science, Springer Verlag, pp. 16-30.

[22] Goldreich, O., Micali, S., ANd Wigderson, A. Proofs that yield nothing but their validity or all languages in $\mathrm{np}$ have zero-knowledge proof systems. Journal of the ACM (JACM) 38, 3 (1991), 690-728.

[23] Goldwasser, S., Micali, S., And Rivest, R. A digital signature scheme secure against adaptive chosen-message attacks. SIAM Journal on Computing 17, 2 (Apr. 1988), 281-308.

[24] Goodrich, M. T., Papamanthou, C., Nguyen, D., TAMASSiA, R., LOPES, C. V., OHRIMENKO, O., AND TRIANDOPOULOS, N. Efficient verification of web-content searching through authenticated web crawlers. Proceedings of the VLDB Endowment 5, 10 (2012), 920-931.

[25] Goodrich, M. T., TAMassia, R., AND TRIANDOPOULOS, N. Efficient authenticated data structures for graph connectivity and geometric search problems. Algorithmica 60, 3 (2011), 505-552.

[26] Gross, T. Certification and efficient proofs of committed topology graphs. Cryptology ePrint Archive Report 2014/255, IACR, 2014. http: / /eprint. iacr.org/.

[27] IBM. Specification of the Identity Mixer cryptographic library, v. 2.3.40. Specification, IBM Research, Jan. 2013. http://prime.inf.tu-dresden.de/idemix/.

[28] Johnson, R., Molnar, D., Song, D., And Wagner, D. Homomorphic signature schemes. In Topics in Cryptology-CT-RSA 2002. Springer, 2002, pp. 244-262.

[29] MiCAli, S., AND Rivest, R. L. Transitive signature schemes. In Topics in Cryptology-CT-RSA 2002. Springer, 2002, pp. 236-243.

[30] Parno, B., Howell, J., Gentry, C., And Raykova, M. Pinocchio: Nearly practical verifiable computation. In 2013 IEEE Symposium on Security and Privacy (SP) (2013), IEEE, pp. 238-252.

[31] Pedersen, T. P. Non-interactive and information-theoretic secure verifiable secret sharing. In Advances in CryptologyCRYPTO '91 (1992), J. Feigenbaum, Ed., vol. 576 of Lecture Notes in Computer Science, Springer Verlag, pp. 129-140.

[32] Peng, K., Boyd, C., And Dawson, E. Batch zero-knowledge proof and verification and its applications. ACM Transactions on Information and System Security (TISSEC) 10, 2 (2007), 6.

[33] Rivest, R. L., Shamir, A., And Adleman, L. A method for obtaining digital signatures and public-key cryptosystems. Communications of the ACM 21, 2 (Feb. 1978), 120-126.

[34] Schiffman, J., Sun, Y., ViJayakumar, H., AND JAEGER, T. Cloud verifier: Verifiable auditing service for iaas clouds. In Services (SERVICES), 2013 IEEE Ninth World Congress on (June 2013), pp. 239-246.

[35] SCHNORR, C. P. Efficient signature generation for smart cards. Journal of Cryptology 4, 3 (1991), 239-252.

[36] SHoup, V. A Computational Introduction to Number Theory and Algebra (Second Edition). Cambridge University Press, 2008. Online http: //www. shoup. net/ntb/.

[37] Xiao, H., Ford, B., And Feigenbaum, J. Structural cloud audits that protect private information. In Proceedings of the 2013 ACM Workshop on Cloud Computing Security Workshop (New York, NY, USA, 2013), CCSW'13, ACM, pp. 101-112.

[38] Zhai, E., Wolinsky, D. I., XiaO, H., LiU, H., Su, X., AND FORD, B. Auditing the structural reliability of the clouds. Tech. rep., YALEU/DCS/TR-1479, Department of Computer Science, Yale University, 2013., 2013.

[39] Zhang, Y., Papamanthou, C., And Katz, J. ALITHEIA: Towards practical verifiable graph processing. In 21st ACM Conference on Computer and Communications Security (ACM CCS'14) (Nov. 2014), ACM Press. 